\title{
Novel red fluorophores with superior performance in STED microscopy
}

\author{
Christian A Wurm ${ }^{1,2^{*}}$, Kirill Kolmakov ${ }^{1}$, Fabian Göttfert ${ }^{1}$, Haisen $\mathrm{Ta}^{1}{ }^{1}$, Mariano Bossi ${ }^{3}$, Heiko Schill ${ }^{1}$, \\ Sebastian Berning ${ }^{1}$, Stefan Jakobs ${ }^{1,2}$, Gerald Donnert ${ }^{1}$, Vladimir N Belov ${ }^{1 *}$ and Stefan W Hell ${ }^{1 *}$
}

\begin{abstract}
In optical microscopy, most red-emitting dyes provide only moderate performance due to unspecific binding, poor labeling efficiency, and insufficient brightness. Here we report on four novel red fluororescent dyes, including the first phosphorylated dye, created by combining a rigidized rhodamine backbone with various polar groups. They exhibit large fluorescence quantum yields and improved NHS ester stability. While these fluorophores are highly suitable for fluorescence microscopy in general, they excel in stimulated emission depletion (STED) microscopy, providing $<25 \mathrm{~nm}$ spatial resolution in raw images of cells.
\end{abstract}

Keywords: Microscopy, Confocal, STED, Fluorescence, Rhodamines, Bioconjugation, Super-resolution, Nanoscopy, Stimulated emission depletion

\section{Introduction}

Red fluorescent labels are particularly useful in life sciences, especially in biological fluorescence microscopy and single molecule spectroscopy (Fu et al. 2008; Bruyneel et al. 2010), because they require excitation photons of lower energy thus entailing less background due to autofluorescence. In addition, they are particularly attractive for stimulated emission depletion (STED) microscopy (Hell and Wichmann 1994; Klar et al. 2000; Dyba et al. 2003) because their fluorescence can be stopped with near-infrared wavelengths (typically $>750 \mathrm{~nm}$ ) that are not only relatively benign to the sample, but also leave the visible spectrum open for the detection of other fluorophores. Therefore, optimizing the brightness, stability and solubility of red fluorescent dyes directly translates into improved performance in all aforementioned fluorescence microscopy modes and STED microscopy in particular.

Because of their good photophysical properties, rhodamine dyes represent an excellent scaffold for the synthesis of improved red fluororescent dyes. One of the most

\footnotetext{
*Correspondence: cwurm@gwdg.de; vbelov@gwdg.de; shell@gwdg.de ${ }^{1}$ Department of NanoBiophotonics, Max Planck Institute for Biophysical Chemistry, Am Fassberg 11, Göttingen 37077, Germany

${ }^{2}$ Department of Neurology, Georg-August University of Göttingen Medical School, Göttingen 37073, Germany

Full list of author information is available at the end of the article
}

frequently used red emitting dyes for single molecule, confocal, and STED microscopy has been Atto647N (Willig et al. 2007; Eggeling et al. 2009). With its large extinction coefficient and high quantum yield, this fluorophore enabled many early STED microscopy studies. Despite these merits, the image contrast was often only modest, which is most likely caused by the lipophilic character of this dye, its low solubility in water, and the resulting unspecific binding to cellular membranes. Also, the presence of two diastereomers may pose problems in analyses like gel electrophoresis or high-performance liquid chromatography (HPLC).

Recently we reported on the red-emitting rhodamine dye KK114 (Figure 1), a bright, photostable, and highly water-soluble fluorescent marker for biomolecules. While KK114 was successfully applied in confocal and STED microscopy (Bückers et al. 2011; Hua et al. 2011; Wurm et al. 2011), the disadvantage of this compound is the negative net charge of the dye residue (making dye conjugates tentatively not cell-permeable), and the moderate hydrolytic stability of the NHS ester. Other dyes, e.g. Cy5 and Alexa647, have been considered but their photostability cannot match the properties of the above dyes under STED imaging conditions. Hence, Atto647N and KK114 set the benchmark for red-emitting, photostable fluorophores both in confocal and STED microscopy. 


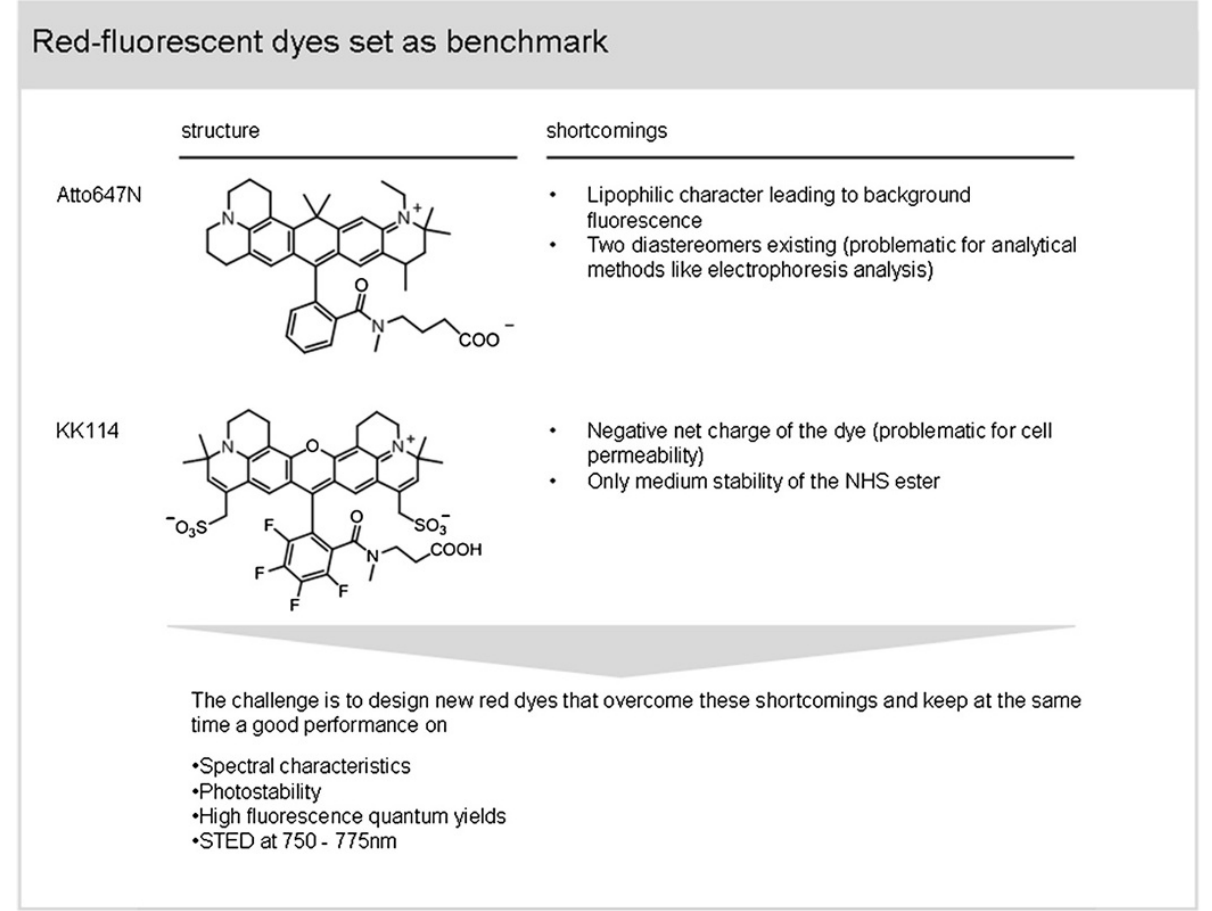

Figure 1 Red fluorescent dyes set the benchmark.

In addition to showing the reversible fluorescence offswitching by STED, the new dyes have to fulfill the following criteria: optimal positions of absorption and emission maxima, high brightness, good photostability under excitation around 620-650 $\mathrm{nm}$ and STED in the range of 750-775 $\mathrm{nm}$. Equally important features of the dyes are hydrophilic character, stability of amino reactive derivatives (e. g., NHS esters), and stability of bioconjugates. To overcome the limitations of the currently widely used dyes, we designed molecules with a rigidized rhodamine fluorophore which is known for its good photophysical properties (Kolmakov et al. 2010). Here we describe the properties of these novel red fluorescent dyes and their performance in STED microscopy.

\section{Results}

\section{Structure and properties of the new red dyes}

The stability of a dye's NHS-ester in aqueous solutions influences not only the handling, but also the yield and reproducibility of reactions with biomolecules such as proteins in bioconjugation procedures. In an effort to improve the stability of the NHS esters, an analog of the dye KK114 with a longer linker between the dye core and the NHS moiety was prepared, shown as KK1119 in Figure 2. The elongation of the linker from 2 to 3 carbon atoms (visible in the structures in Figures 1 and 2) increased the hydrolytic stability of the corresponding
NHS esters, while maintaining the favorable properties of KK114.

Additionally, both variable positions ( $\mathrm{R}$ and $\mathrm{X}$ ) in the rhodamine backbone were utilized to obtain compounds with improved properties (Figure 2). Particularly, hydroxylation of two methyl groups (at position R) attached to the $\mathrm{C}=\mathrm{C}$ double bonds converted them into the corresponding allylic alcohols $(\mathrm{R}=\mathrm{OH})$. This transformation provided compound KK9046, without introducing an additional negative charge inhibiting cell-permeability.

To further improve the water solubility of the hydroxyl substituted dye (KK9046), the second variable position (X) was involved. One of the four fluorine atoms in the aromatic ring was selectively exchanged with a 2mercaptoethyl sulfonic acid residue $\left(\mathrm{X}=\mathrm{S}\left(\mathrm{CH}_{2}\right)_{2} \mathrm{SO}_{3} \mathrm{H}\right.$; Figure 2). This modification had only minor effect on the spectral properties of the dye and provided an "amphiphilic dye", called Abberior STAR635, with good performance in imaging and a very stable NHS-ester. Finally, the hydroxymethyl residues in the rhodamine KK9046 were transformed into primary phosphate groups $\left(\mathrm{R}=\mathrm{OP}(\mathrm{O})(\mathrm{OH})_{2}\right)$ providing a new dye, dubbed STAR635P. To our knowledge, this is the first phosphorylated rhodamine representing a new alternative to the widely used sulfonated fluorophores. High polarity and the increased number of acidic protons (relative to sulfonic acid residues) in the phosphorylated dye 
STAR635P provide good solubility in neutral and basic aqueous solutions ( $\mathrm{pH}>7$ ) accompanied by a large fluorescence quantum yield.

While the syntheses of the new dyes and all the related data will be detailed elsewhere (Kolmakov et al. 2012), Figure 2 shows the properties of the new red-emitting compounds and the benchmark dyes Atto647N and KK114. The required spectral properties and fluorescence quantum yields of the new dyes in solution are similar to the benchmark dyes. In antibody conjugates, the fluorescence quantum yields were found to be high (up to $48 \%$ ). These are, to our knowledge, the highest values that have so far been reported for near-IR emitting dye conjugates with proteins. The net charges of the new dyes were varied; in conjugates of KK9046 and STAR635 they are positive and zero, respectively, which is favorable for cell permeability (Kolmakov, et al. 2012). As expected, the polar groups - sulfonic acid, hydroxyl and primary phosphate residues - increase the solubility in water and aqueous buffers relative to Atto647N. In 

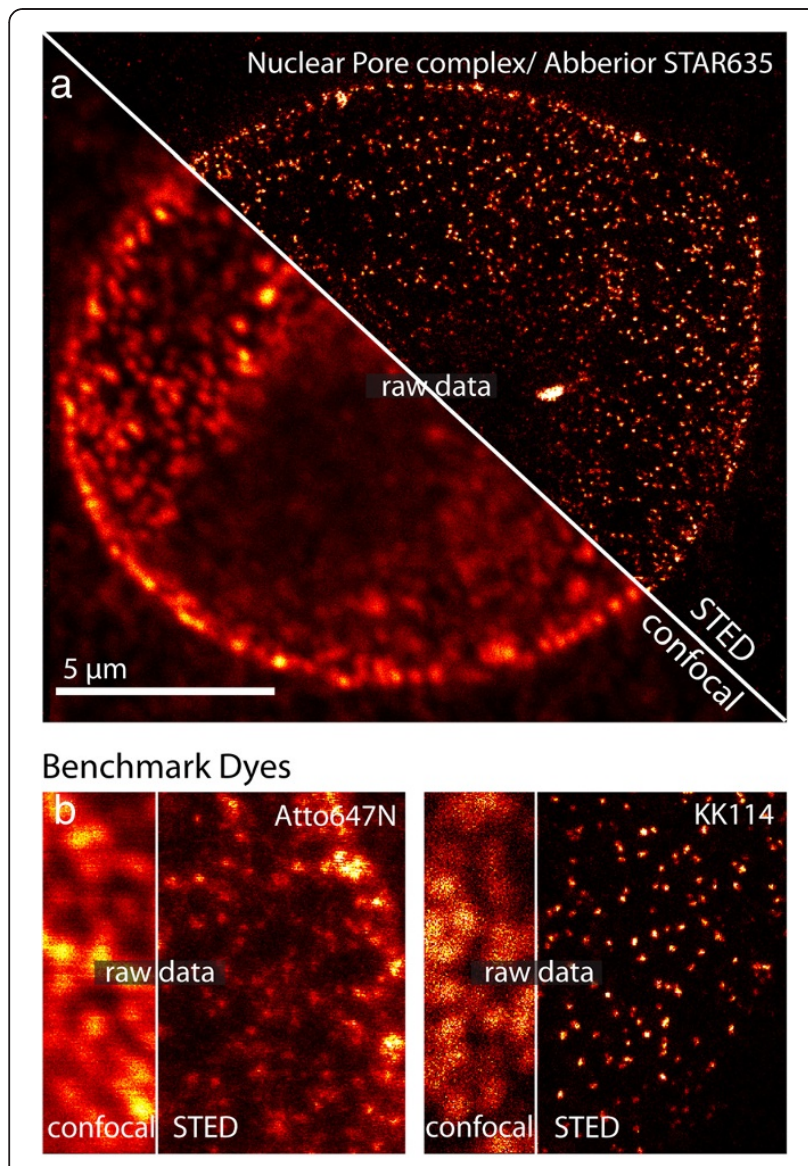

\section{Novel red dyes}
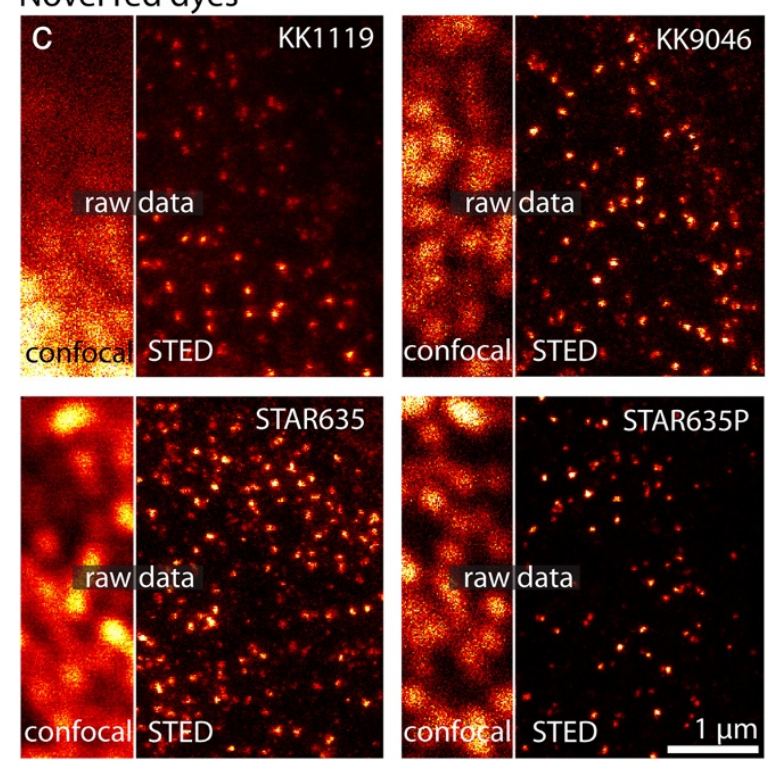

Figure 3 Comparison of the performance of the novel red dyes with the benchmark dyes Atto647N and KK114. (a) Overview image of the nucleus of a fixed PtK2 cell showing the Nup153 subunit of the nuclear pore complex immunolabeled with the different dyes coupled to secondary antibodies. Confocal microscopy (left) fails to discriminate individual nuclear pore complexes, whereas STED microscopy (right) identifies essentially each individual complex. (b) Comparison of confocal and STED recordings of the benchmark dyes Atto647N and KK114. Similar comparison between the novel red dyes in (c). Only subdiffraction resolution microscopy enables the accurate size determination (between 40 and $60 \mathrm{~nm}$ ) of the labeled structures. The novel dye conjugates KK1119, KK9046, STAR635 and STAR635P display superior contrast and less background labeling than Atto647N. All data is raw.

addition, we found that the elongation of the linker between the fluorophor and NHS moiety led to an increased stability of the NHS esters compared to KK114. The fluorescence lifetime of the free dyes and antibody conjugates in solution was found to be comparable for the benchmark dyes and the new dyes (Figure 2 \& additional file). In cell samples the lifetimes were found to be different, though. The shortest lifetimes were $1.2 \mathrm{~ns}$ for Atto647N and KK1119, whereas the longest ones were well above 3 ns for KK114 and STAR635P (Figure 2, Additional file 1: Figure S2).

\section{STED imaging performance of new red dyes}

For STED microscopy and the corresponding confocal microscopy, either a stage scanning STED microscope (Exc. $635 \mathrm{~nm}$; STED $775 \mathrm{~nm} / 20 \mathrm{MHz} / 1 \mathrm{~ns}$ pulses from frequency-doubled fiber laser; Detection range 670/ $40 \mathrm{~nm}$ ) or a beam scanning STED microscope (Exc. $640 \mathrm{~nm}$; STED $760 \mathrm{~nm} / 76 \mathrm{MHz} / \sim 200$ ps pulses upchirped from 100 fs mode-locked Ti: Sapphire laser; Detection range $670 / 40 \mathrm{~nm}$ ) was used. The cultivation of cells, antibody and immunolabeling was performed as described elsewhere (Wurm et al. 2010). For further details on imaging and sample preparation, please refer to the additional file.

We found that all new dyes are especially suitable for immunofluorescence assays and imaging (Figure 3). The confocal images are bright, demonstrating high signal-to-noise ratios while their STED counterparts provide, in addition, outstanding spatial resolution. Atto647N and STAR635 yield the brightest STED images. Another important parameter associated with brightness is the staining contrast, i.e. the absence of a fluorescent background stemming from unspecific staining. In this respect, STAR635P performs best, as it produces practically no background. The performance of the two hydroxylated dyes in imaging - KK9046 and STAR635 - is virtually the same, which is in good agreement with the similarity of their photophysical 

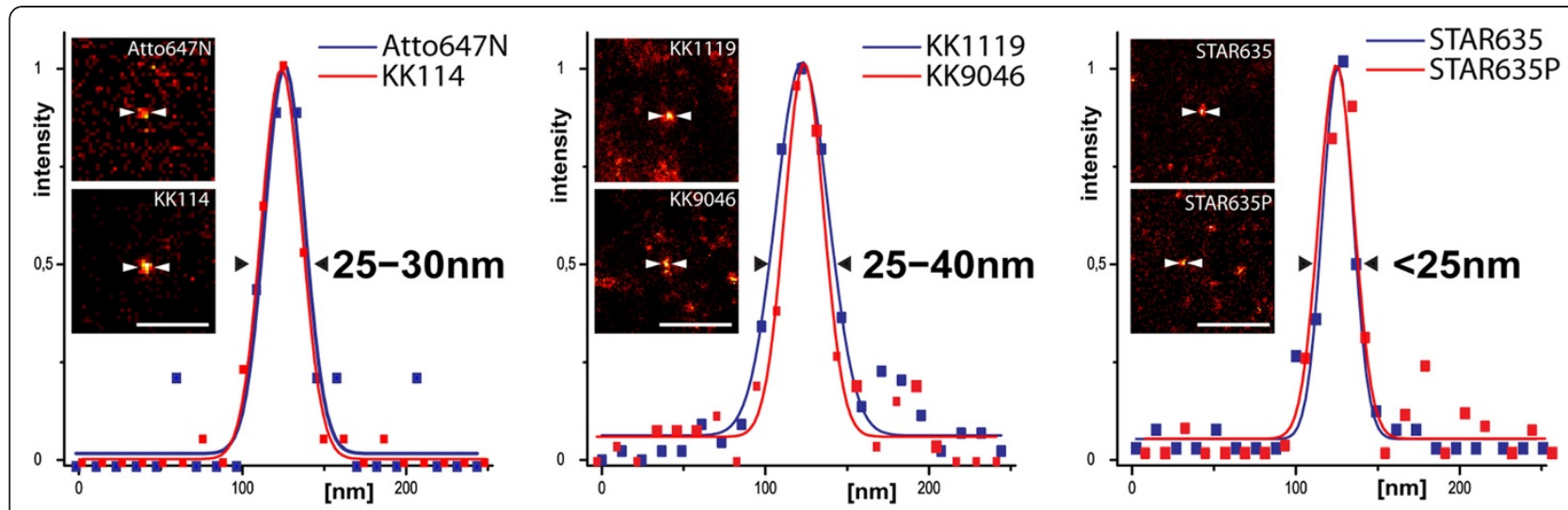

Figure 4 Spatial resolution with the benchmark dyes and the four novel red dyes in the focal plane of a STED microscope. The insert panels show the individual 'point-like' raw data spots (most likely antibody clusters) from which the shown line profiles were extracted, indicating the spatial resolution in the focal plane. The solid lines represent optimal Gaussian fit curves. The novel STAR dyes provides a focal plane resolution of $<25 \mathrm{~nm}$ (in raw data). Scale bars, $500 \mathrm{~nm}$.

properties. Having a sulfonic acid group, STAR635 has superior solubility in water, and is even better soluble in aqueous $\mathrm{NaHCO}_{3}$ and/or PBS buffer solutions (compared to KK9046).

Recording STED images of the Nup153 subunit of the nuclear pore complex (NPC) in cultured mammalian cells, we found this subunit (which is distantly located in the nuclear basket) to form clusters with a diameter of 40-60 nm (Additional file 1: Figure S3). This finding is in line with previous electron microscopy data (Fahrenkrog et al. 2002).

To quantify the spatial resolution that is achieved with the different dyes, we identified isolated bright point-like fluorescent objects, most likely due to individual antibody clusters (Figure 4). Evaluating their profile in the focal plane revealed a resolution of $230 \mathrm{~nm}$ in the confocal case, while in the STED case a resolution of $<25 \mathrm{~nm}$ was achieved. As a result, the combination of fluorophore, pulse wavelength and duration provided an $\sim 9$-fold increase in resolution. Note that this resolution is attained in raw data and hence exclusively based on optically induced molecular transitions.

The imaging performance with STAR635P was further evaluated by directly comparing Atto647N and STAR635P on immunolabeled tubulin fibers in mammalian cells (Figure 5). The images consistently prove the severely reduced background signal in the STAR635P staining. All labeling procedures were optimized for each individual dye. Figure 6 compares the confocal and STED images of immunofluorescence labeled Vimentin using STAR635P. Note the superior contrast and resolution attained in raw data.

\section{Conclusion and outlook}

The novel red fluorescent dyes investigated in this study afford superior image brightness and contrast owing to the low background and large fluorescence quantum yields. Moreover, they enable a spatial resolution down to $25 \mathrm{~nm}$ in raw STED microscopy data. We also identified phosphorylated fluorophores as alternatives to the traditional sulfonated counterparts which show reduced unspecific background fluorescence. This finding may form the basis of a new family of fluorescent compounds providing unparalleled properties not only for far-field

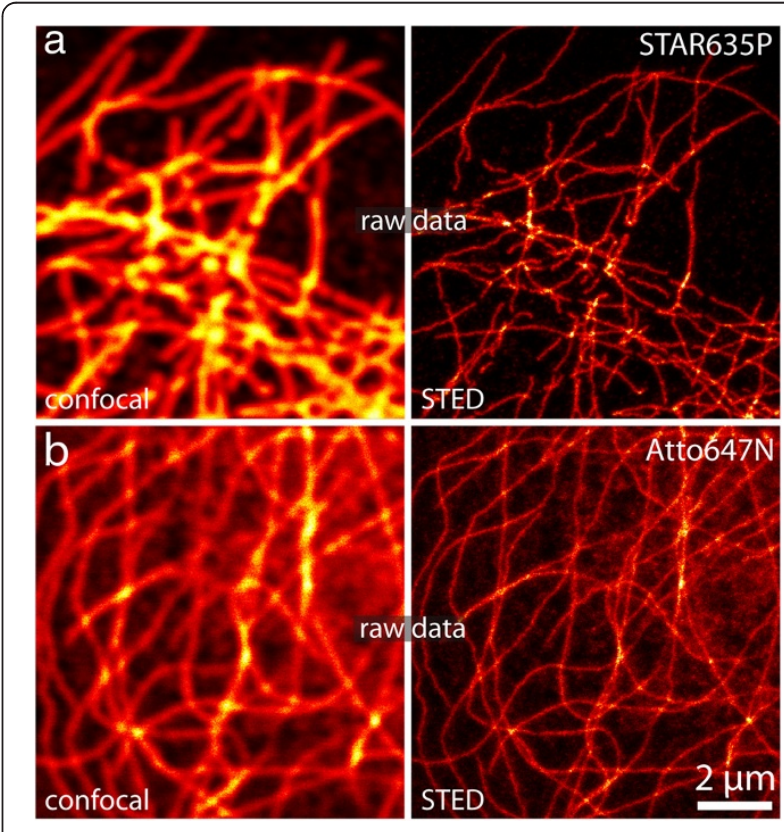

Figure 5 Comparison of the novel dye STAR635P (a) and the benchmark dye Atto647N. (b) The tubulin cytoskeleton was immunolabeled in fixed PtK2 cells and imaging of the same area in the cell performed in the confocal and STED modes. Note the absence of virtually any background with the STAR635P staining. Images display raw data. Imaging was performed using a beam scanning STED microscope equipped with a Ti:Sapphire laser. 


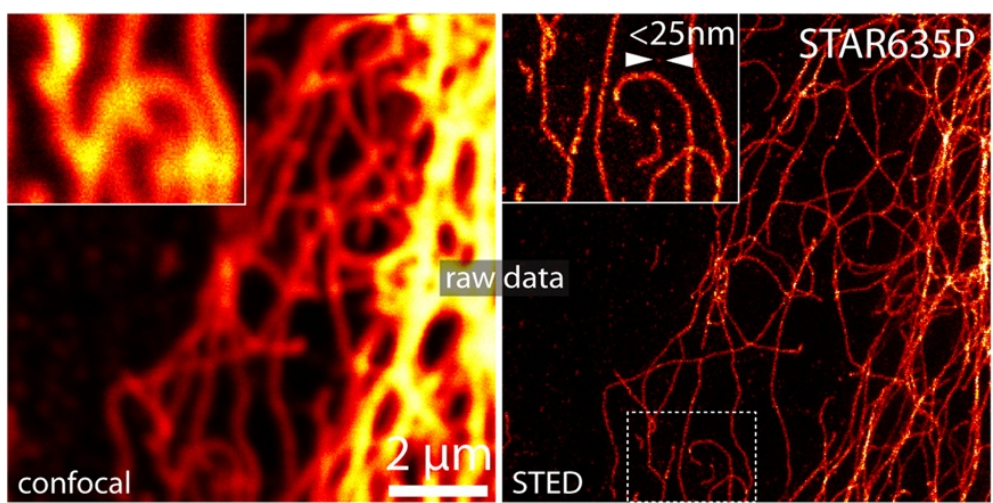

Figure 6 Resolution gain by STED over confocal microscopy in raw data. Confocal (left) and STED (right) imaging of Abberior STAR635P labeled Vimentin. Note the optical resolution $<25 \mathrm{~nm}$ identified with an individual antibody cluster in the STED image (arrows in the inset).

optical nanoscopy, but all applications of fluorescence labeling, including single molecule studies and (bio)analytical chemistry.

\section{Additional files}

Additional file 1: Figure S1. Absorption and emission spectra of the benchmark dyes ATTO647N and KK114. Additional file 1: Figure S2. Fluorescence decay and lifetime of the red dyes in cell samples (PtK2 cells; vimentin labeling): curves fitting the spontaneous decay of the fluorescent state. Note that the slope of the curves varies for the different dyes, resulting in fluorescence lifetimes ranging from $1.2 \mathrm{~ns}$ to $3.6 \mathrm{~ns}$. Additional file 1: Figure S3. Measurements of the size of nuclear pore complexes. After immunolabeling with the different fluorophores and imaging with super-resolution STED microscopy, the average diameter of the nuclear pore complexes was determined $(n>5)$. Using fluorophores with improved solubility in aqueous buffers, smaller average sizes of the nuclear pore complexes were determined. This is most likely due to the superior signal-to-noise ratio in the images. Supplemental Methods and Results: (Enderlein and Erdmann 1997), (Fery-Forgues and Lavabre 1999).

\section{Competing interests}

Some of the authors (GD, VNB, and SWH) hold shares of Abberior $\mathrm{GmbH}$, Göttingen, Germany distributing two of the dyes investigated in this paper. The authors CAW, MB, SB, FG, SJ, KK, HS, HT declare that they have no competing interests.

\section{Authors' contributions}

GD, CAW, SJ, VNB, SWH designed research and wrote the manuscript; KK and VNB designed and performed the chemical synthesis; CAW prepared the cell and antibody samples; CAW, FG, SB and HT did the confocal and STED imaging; CA, W, HT, MB, HS carried out the spectroscopic characterization of the novel dyes; All authors read and approved the final manuscript.

\section{Acknowledgements}

The authors are grateful to Nina Ohm and Marianne Pulst for recording the optical spectra. We also thank Ellen Rothermel for excellent technical assistance and Jaydev Jethwa for critical reading of the manuscript. MB is research staff from CONICET. We are indebted to the Bundesministerium für Bildung und Forschung (BMBF 513) for financial support in the program Optische Technologien für Biowissenschaften und Gesundheit (FKZ 13N11066) and for support by the DFG-Research Center for Molecular Physiology of the Brain.

\section{Author details}

'Department of NanoBiophotonics, Max Planck Institute for Biophysical Chemistry, Am Fassberg 11, Göttingen 37077, Germany. ${ }^{2}$ Department of
Neurology, Georg-August University of Göttingen Medical School, Göttingen 37073, Germany. ${ }^{3}$ Inquimae Conicet-UBA, Pabellon 2, Ciudad Universitaria, Buenos Aires, Argentina.

Received: 12 July 2012 Accepted: 11 September 2012 Published: 28 September 2012

\section{References}

Bruyneel F, D'Auria L, Payen O, Courtoy PJ, Marchand-Brynaert J (2010) Live-cell imaging with water-soluble aminophenoxazinone dyes synthesised through laccase biocatalysis. ChemBioChem 11(10):1451-1457

Bückers J, Wildanger D, Vicidomini G, Kastrup L, Hell SW (2011) Simultaneous multi-lifetime multi-color STED imaging for colocalization analyses. Optics Express 19(4):3130-3143

Dyba M, Jakobs S, Hell SW (2003) Immunofluorescence stimulated emission depletion microscopy. Nature Biotechnology 21(11):1303-1304

Eggeling C, Ringemann C, Medda R, Schwarzmann G, Sandhoff K, Polyakova S, Belov VN, Hein B, von Middendorff C, Schonle A, Hell SW (2009) Direct observation of the nanoscale dynamics of membrane lipids in a living cell. Nature 457:1159-U1121

Enderlein J, Erdmann R (1997) Fast fitting of multi-exponential decay curves. Optics Communications 134(1-6):371-378

Fahrenkrog B, Maco B, Fager AM, Koser J, Sauder U, Ullman KS, Aebi U (2002) Domain-specific antibodies reveal multiple-site topology of Nup153 within the nuclear pore complex. Journal of Structural Biology 140(1-3):254-267

Fery-Forgues S, Lavabre D (1999) Are Fluorescence Quantum Yields So Tricky to Measure? A Demonstration Using Familiar Stationery Products. Journal of Chemical Education 76(9):1260

Fu M, Xiao Y, Qian X, Zhao D, Xu Y (2008) A design concept of long-wavelength fluorescent analogs of rhodamine dyes: replacement of oxygen with silicon atom. Chemical Communications 15:1780-1782

Hell SW, Wichmann J (1994) Breaking the diffraction resolution limit by stimulated-emission - stimulated-emission-depletion fluorescence microscopy. Optics Letters 19(11):780-782

Hua Y, Sinha R, Thiel CS, Schmidt R, Hüve J, Martens H, Hell SW, Egner A, Klingauf J (2011) A readily retrievable pool of synaptic vesicles. Nature Neuroscience 14(7):833-839

Klar TA, Jakobs S, Dyba M, Egner A, Hell SW (2000) Fluorescence microscopy with diffraction resolution barrier broken by stimulated emission. Proceedings of the National Academy of Sciences of the United States of America 97:8206-8210

Kolmakov K, Belov V, Bierwagen J, Ringemann C, Mueller V, Eggeling C, Hell S (2010) Red-emitting rhodamine dyes for fluorescence microscopy and nanoscopy. Chemistry: A European Journal 16(1):158-166

Kolmakov K, Wurm CA, Hennig R, Rapp E, Jakobs S, Belov VN, Hell SW (2012) Red-emitting rhodamines with hydroxylated, sulfonated, and phosphorylated dye residues and their use in fluorescence super-resolution microscopy. Chemistry: A European Journal 18(14):986-98.

Willig Kl, Harke B, Medda R, Hell SW (2007) STED microscopy with continuous wave beams. Nature Methods 4(11):915-918 
Wurm CA, Neumann D, Lauterbach MA, Harke B, Egner A, Hell SW, Jakobs S

(2011) Nanoscale distribution of a mitochondrial import receptor Tom20 is adjusted to cellular conditions and exhibits an inner-cellular gradient. Proceedings of the National Academy of Sciences of the United States of America 108(33):13546-13551

Wurm CA, Neumann D, Schmidt R, Egner A, Jakobs S (2010) Sample preparation for STED microscopy. Methods in Molecular Biology 591:185-199

doi:10.1186/2192-2853-1-7

Cite this article as: Wurm et al: Novel red fluorophores with superior performance in STED microscopy. Optical Nanoscopy 2012 1:7.

Submit your manuscript to a SpringerOpen ${ }^{\circ}$ journal and benefit from:

- Convenient online submission

- Rigorous peer review

- Immediate publication on acceptance

- Open access: articles freely available online

- High visibility within the field

- Retaining the copyright to your article

Submit your next manuscript at $>$ springeropen.com 